\title{
A Note on the History of the Gambler's Ruin Problem
}

\author{
Seongjoo Song ${ }^{a}$, Jongwoo Song ${ }^{1, b}$ \\ ${ }^{a}$ Department of Statistics, Korea University; ${ }^{b}$ Department of Statistics, Ewha Womans University
}

\begin{abstract}
This paper deals with the history of one of the well-known and historically important problems in probability, "Gambler's ruin". This problem was first solved by Pascal and Fermat and published by Huygens in 1657. It was studied and extended by many probabilists in early years and thus, it became an important problem in probability history, introducing many new concepts. We would like to introduce the problem in detail to readers and share the ideas on how new problems are developed, relating to old problems.
\end{abstract}

Keywords: Gambler's ruin, Huygens' fifth problem.

\section{Introduction}

Suppose a stock market investor owns shares in a stock whose present value is 25 . He decided to sell his stock if it either goes down to 10 or up to 40 . If each change of stock price is either up 1 point with probability $p$ or down 1 point with probability $q$, and the successive changes are independent, what is the probability that the investor retires a winner? (Ross, 1997) Although this example may be too simple to be real, we can find situations in investment that can be simplified to similar cases. This is, in fact, an illustration of a well-known problem in probability, known as "Gambler's ruin".

The Gambler's ruin problem was proposed by Pascal to Fermat in 1656. It is two years later than the correspondence between Pascal and Fermat on the "Problem of points". It is not hard to imagine that it was an interesting and difficult problem among gamblers at that time, because we can see similar situations in the modern finance. These days people are interested in various probabilities related to many financial instruments. In particular, when people make up a new financial instrument, such as a new type of stock option, they calculate many types of probabilities that can show how profitable it is. Presumably, Pascal did not have any practical concern about this problem, but he considered it as a very difficult and interesting problem in the mathematical perspective.

The two mathematicians, Pascal and Fermat, solved this problem independently and presumably with different methods. In the same year, it was handed over to a dutch mathematician, Huygens through a French amateur mathematician, Carcavi, who was a friend of Fermat's. By Ore (1960), Pascal gave Carcavi this problem to transmit to Huygens after he saw Huygens' first work on the probability. In 1657, Huygens published his 16-page-treatise, De Ratiociniis in Ludo Aleae, which is the first published work on probability theory, and there he introduced the Gambler's ruin problem with 4 other problems to common readers. So the Gambler's ruin problem is also known as Huygens' fifth problem. It played a very important role in the development of probability theory with the other 4 problems. Many mathematicians tried to solve and extend this problem in various ways. Meanwhile, it results in a beginning of the theory of random walks, Brownian Motion, and more applications.

This research was supported by the Basic Science Research Program through the National Research Foundation of Korea (NRF) funded by the Ministry of Education, Science and Technology (No. 2012-0003739(J.Song) and No. 20120006923(S.Song)).

${ }^{1}$ Corresponding author: Associate professor, Department of Statistics, Ewha Womans University, 11-1 Daehyun-dong, Seodaemun-gu, Seoul 120-750, Korea. E-mail: josong@ewha.ac.kr 


\section{Description of the Problem}

\subsection{Carcavi's description (Edwards, 1987)}

Let two men play with three dice, the first player scoring a point whenever 11 is thrown, and the second whenever 14 is thrown. But instead of the points accumulating in the ordinary way, let a point be added to a player's score only if his opponent's score is zero, but otherwise let it instead be subtracted from his opponent's score, so that the trailing player always has zero points. The winner is the first to reach twelve points; what are the relative chances of each player winning?

\subsection{Huygens' description (Edwards, 1987)}

1. First formulation of the problem: The player A scoring a point whenever 11 is thrown, and the player B whenever 14 is thrown. The points accumulate in the ordinary way, but the winner is the one who first leads by twelve points.

2. When he wrote 'De ratiociniis in ludo aleae'(Huygens, 1657): Two players start with twelve points. If the player A wins, then a point of B's is transferred to A. The winner is the one who bankrupts the other of points.

The second formulation is the way that Gambler's ruin problem is introduced to common readers and the first formulation is what Huygens used in his proof. These two and Carcavi's formulations are equivalent.

\subsection{General description by James Bernoulli and De Moivre (Hald, 1990)}

Let A have $m$ counters and B $n$ counters at the beginning of the play, and let their probabilities of winning in each trial be $p$ and $q=1-p$, respectively. After each trial the winner gets a counter from the loser, and the play continues until one of the players is ruined. What is the probability of A being ruined? In Huygens' second description, $p=9 / 14, q=5 / 14$, and $m=n=12$.

\subsection{Modern description (Feller, 1970)}

Consider a familiar gambler who wins or loses $a$ dollar with probabilities $p$ and $q$, respectively. He has the initial capital, $z$, and plays against an opponent with the initial capital, $a-z$. The game continues until the gambler's capital either is reduced to zero or has increased to $a$, that is, until one of the two players is ruined. What is the probability of the gambler's ruin?

The problem may also be described as a random walk starting at $0<z<a$ with absorbing barriers at 0 and $a$. Consider a particle on the $x$-axis. It starts at $z$ and it moves to the right or left with probability $p$ and $q$, respectively. The trial terminates when the particle reaches either 0 or $a$. The position of the particle after $n$ steps represents the gambler's capital after $n$ games. It can be considered as a crude approximation to one dimensional diffusion or Brownian Motion.

\section{Pascal's Solution}

The solution that Pascal gave to Carcavi without mentioning the method of solution was

$150,094,635,296,999,122: 129,746,337,890,625$. 
In terms of probability, the probability that the player A wins ultimately is:

$$
\frac{150,094,635,296,999,122}{150,094,635,296,999,122+129,746,337,890,625}=.999136316,
$$

when the odds of the player A's scoring versus B's scoring are $27: 15$. Since the probability of A's winning at a single play is $27 / 42$ and it is almost the double of the probability of B's winning which is $15 / 42$, it is plausible that the probability of A's ultimate winning is such a big number. Although there is no way to find Pascal's actual reasoning for the odds, it is sensible that we find his method of solution in the earlier work on the "Problem of points". Edwards (1987) gives a conjecture on how Pascal came up with the above solution using his "old" method of recursive formula used in solving the "Problem of points". Edwards' explanation is as follows.

Pascal would use the notion of the expectation and the recursive formula as he did earlier. Define $E(a, b)$ to be the expected winnings of player A when A has $a$ points and $\mathrm{B}$ has $b$ points. Let us use Carcavi's description of the problem. Then he would have

$$
E(a, b)=p E(a+1, b)+q E(a, b+1),
$$

with $E(12,0)=1, E(0,12)=0$. Here the total stake is assumed to be 1 so that the expectation of $\mathrm{A}$ is the same as the probability of A's winning. Now, change the scale of $(12,0)$ to $(0,12)$ to the scale of (0) to (24). Then $E(0,12)=E_{0}=0, E(0,11)=E_{1}, E(0,10)=E_{2}, \ldots, E(0,0)=E_{12}$, $E(1,0)=E_{13}, \ldots$, and $E(12,0)=E_{24}=1$. From (3.2), Pascal would have

$$
E_{i}=p E_{i+1}+q E_{i-1}
$$

for $i=1, \ldots, 23$, with $E_{0}=0, E_{24}=1$. From this, we get $p E_{i+1}-p E_{i}=q E_{i}-q E_{i-1}$, for $i=$ $1, \ldots, m-1$. Add these $m-1$ equations altogether to get $p E_{m}-p E_{1}=q E_{m-1}$ since $E_{0}=0$. By some substitutions, we get

$$
E_{m}=\frac{1-\left(\frac{q}{p}\right)^{m}}{1-\frac{q}{p}} E_{1},
$$

for $m=1, \ldots, 24$. Since $E_{24}=1, E_{1}$ is easily obtained by putting $m=24$ in the above equation. Therefore, the desired probability, which is the player A's winning probability, is

$$
E_{12}=E(0,0)=\frac{1-\left(\frac{q}{p}\right)^{12}}{1-\left(\frac{q}{p}\right)^{24}} .
$$

Similarly, the player B's winning probability (A's ruin probability) is given by (3.3) with $p$ and $q$ exchanged. Therefore, the odds of the player A's winning versus B's winning is $p^{12} / q^{12}$. If we put $p=27$ and $q=15$, then we get the odds given in (3.1).

\section{Fermat's Solution}

Right after Fermat heard about this problem from Pascal, he promptly returned a correct solution to Pascal through Carcavi. According to him, the odds of the player A's winning versus B's winning are between $1156: 1$ and $1157: 1$. Simply dividing $150,094,635,296,999,122$ by $129,746,337,890,625$, 
we can see that Pascal's solution gives the odds equal to $1156.83: 1$. Although Fermat did not explain how he ended up with the above odds, it seems that he used a correct and maybe approximate method to solve the problem, from the fact that his solution almost agreed with Pascal's. Pascal did not seem to believe that Fermat actually solved it because he thought this problem is too difficult to solve so quickly even for Fermat. But we may guess how Fermat thought of this problem from the way he solved the "Problem of points". Edwards (1987) also gives a suggestion on the argument that Fermat used. By Edwards (1987), Fermat would presumably have used the combinatorial argument as in the "Problem of points". Since the game can continue very long, he would start with the shortest possible case. In the shortest possible game which consists of 12 throws of dice, odds of A's (straight) winning versus B's winning are $p^{12}: q^{12}$, where the odds of A's winning at a single throw versus B's winning are $p: q$. In the next shortest game consisting of 14 throws, odds are $\left(\begin{array}{c}12 \\ 1\end{array}\right) p^{13} q:\left(\begin{array}{c}12 \\ 1\end{array}\right) p q^{13}=$ $p^{12}: q^{12}$. By symmetry, odds are all the same as $p^{12}: q^{12}$. Therefore, the odds for the whole game will be $p^{12}: q^{12}$. When $p=9, q=5$, we get the odds between 1156:1 and 1157:1 that is Fermat's solution. This is much simpler but not as general as Pascal's method. However, it is not very meaningful to compare Fermat's and Pascal's methods at this moment, because those are only conjectures.

\section{Huygens' Solution}

By Hald (1990), after Huygens heard about this problem from Carcavi, he answered back with a solution within 15 days. His odds of the player A's winning versus the player B's winning are $282,429,536,481$ to $244,140,625$. These odds are simpler than Pascal's odds, but we can easily see that these are exactly the same as Pascal's. Indeed, $282,429,536,481 \times 3^{12}=150,094,635,296,999,122$ and $244,140,625 \times 3^{12}=129,746,337,890,625$. Although his method of solution was not included in the letter to Carcavi, it was contained in his note in 1676. In the proof, Huygens used the formulation in which the players start with the null, the points accumulate ordinarily, and the winner is the one who first leads by 12 points. He started with the case where the winner is the one who first leads by 2 points. Denote $e(a, b)$ to be A's expectation when A has $a$ points and B has $b$ points. Assume that the total stake is 1 so that $e(a, b)$ is also the probability of A's winning, which is the same as the probability of the gambler B's ruin. Then, $e(2,0)=1$ and $e(0,2)=0$. Note that the desired probability is $e(0,0)$ which is the probability of A's winning. It is clear that

$$
\begin{aligned}
& e(1,0)=p e(2,0)+q e(1,1)=p+q e(0,0), \\
& e(0,1)=p e(1,1)+q e(0,2)=p e(0,0), \\
& e(0,0)=p e(1,0)+q e(0,1),
\end{aligned}
$$

and therefore, $e(0,0)=p^{2} /\left(p^{2}+q^{2}\right)$. Next, consider the case where a player should lead by 4 points to win. Huygens thought of the two steps as a pair. Similar to the previous case,

$$
\begin{aligned}
& e(2,0)=p^{2} e(4,0)+q^{2} e(2,2)=p+q^{2} e(0,0), \\
& e(0,2)=p^{2} e(2,2)+q^{2} e(0,4)=p^{2} e(0,0), \\
& e(0,0)=p^{2} e(2,0)+q^{2} e(0,2),
\end{aligned}
$$

and therefore, $e(0,0)=p^{4} /\left(p^{4}+q^{4}\right)$. By similar arguments, $e(0,0)=p^{8} /\left(p^{8}+q^{8}\right)$ for the case where 
8-point-lead is needed to win. For the case where the winner is the one who first leads by 3 points,

$$
\begin{aligned}
& e(1,0)=\frac{p^{2} e(3,0)+q^{2} e(1,2)}{p^{2}+q^{2}}=\frac{p^{2}+q^{2} e(0,1)}{p^{2}+q^{2}}, \\
& e(0,1)=\frac{p^{2} e(2,1)+q^{2} e(0,3)}{p^{2}+q^{2}}=\frac{p^{2} e(1,0)}{p^{2}+q^{2}}, \\
& e(0,0)=p e(1,0)+q e(0,1),
\end{aligned}
$$

and therefore, $e(0,0)=p^{3} /\left(p^{3}+q^{3}\right)$. He also generalized it to the case where the player should lead by 6 points. From these, he concluded that $e(0,0)=p^{n} /\left(p^{n}+q^{n}\right)$ in the case where the winner should lead by $n$ points. The general solution for odds of A's winning versus B's winning are $p^{n} /\left(p^{n}+q^{n}\right): q^{n} /\left(p^{n}+q^{n}\right)=p^{n}: q^{n}$. Since $p=9 / 14, q=5 / 14$ and $n=12$, the odds are $9^{12}: 5^{12}=282,429,536,481: 244,140,625$. Even though his proof counts on an incomplete induction, Huygens' solution has an important meaning in the sense that it is the first proof of the problem known to us and it made all the following works possible. See Hald (1990) for more details.

\section{Generalizations}

\subsection{James Bernoulli}

A general form of the Gambler's ruin problem was solved by James Bernoulli and published 8 years after his death in Ars Conjectandi (The Art of Conjecturing) (Bernoulli, 1713). By Hald (1990), in Part I of Ars Conjectandi, he generalized the problem and replaced Huygens' numerical results by formulae. Recall the general description of James Bernoulli in Section 2.3. Denote $P_{A}$ and $P_{B}$ to be the probability of A's winning and B's winning, respectively. Huygens solved for $m=n=1,2,3,4,6$ and concluded that the general solution is $P_{A}: P_{B}=p^{m}: q^{m}$. James Bernoulli gives the solution of the general problems of $m \neq n$ as

$$
P_{A}: P_{B}=\left(p^{n} q^{m}-p^{m+n}\right):\left(q^{m+n}-p^{n} q^{m}\right),
$$

but the proof was left to readers. For the case of $m=n$, he used the general recursion formula such as

$$
e(x)=p e(x+1)+q e(x-1)
$$

where $e(x)$ is A's expectation when he has $x$ counters for $x=1, \ldots, 2 m-1$. Again, the total stake is assumed to be 1 so that $e(x)$ is the same as the probability of A's winning. His proof also counts on an incomplete induction like Huygens' proof. A complete proof of the general problem based on the difference equation was first given by Struyck (1716).

\subsection{De Moivre}

By considering the specific nominal value of each counter, de Moivre solved the Gambler's ruin problem in a very clever and simple way in 1711. By Thatcher (1957), the proof is as follows.

The player A has $a$ counters and B $b$ counters before them in a pile. The nominal values are assigned to the counters in the following manner:

$$
\text { A's bottom counter is given the nominal value } \frac{q}{p}
$$

A's second bottom counter is given the nominal value $\left(\frac{q}{p}\right)^{2}$ 


$$
\begin{aligned}
& \text { A's top counter is given the nominal value }\left(\frac{q}{p}\right)^{a} \\
& \text { B's top counter is given the nominal value }\left(\frac{q}{p}\right)^{a+1} \\
& \text { B's second top counter is given the nominal value }\left(\frac{q}{p}\right)^{a+2} \\
& \text { B's bottom counter is given the nominal value }\left(\frac{q}{p}\right)^{a+b} \text {. }
\end{aligned}
$$

After each game, the loser's top counter is transferred to the top of the winner's pile. In terms of nominal values, B's stake is always $q / p$ times A's, so that each player's nominal expectation is 0 at each game. A's nominal expectation is the same as -B's nominal expectation, which is $p(q / p)^{x+1}-$ $q(q / p)^{x}=0$, for any value of $x$. Therefore,

A's nominal expectation in the whole game = B's nominal expectation

$$
\begin{aligned}
\Longleftrightarrow & \text { A's chance of winning all B's counters } \times \text { A's nominal gain } \\
& =\text { B's chance of winning all A's counters } \times \text { B's nominal gain } \\
\Longleftrightarrow & P_{A}\left\{\left(\frac{q}{p}\right)^{a+1}+\cdots+\left(\frac{q}{p}\right)^{a+b}\right\}=P_{B}\left\{\left(\frac{q}{p}\right)+\cdots+\left(\frac{q}{p}\right)^{a}\right\},
\end{aligned}
$$

where $P_{A}$ and $P_{B}$ are the prbabilities of A's winning and B's winning, respectively. Assuming $P_{A}+$ $P_{B}=1$, the probability that A wins the whole game is,

$$
P_{A}=\frac{1-\left(\frac{q}{p}\right)^{a}}{1-\left(\frac{q}{p}\right)^{a+b}} .
$$

If we plug in $a=b=12$, then we get the exactly the same answer as Pascal's $E_{12}$. And the odds of A's winning versus B's winning are $P_{A} / P_{B}=p^{12} / q^{12}$.

\subsection{Ampère}

The name of André-Marie Ampère is familiar to most people because the unit of electric current is named after him. He was a physicist, but he also worked on some mathematical subjects. In his first mathematics essay, Considérations sur la Théorie mathématique du Jeu (Ampère, 1802), he studied the gambler's ruin probability with a combinatorial argument and power series expansion. His method is quite different from others and is more complicated. Because his approach is very different from others, it is pretty clear that he did not know how other people solved this problem. He considered the probability that a gambler with original fortune, $m$, is eliminated from the game after he wins $p$ games and loses $m+p$ games. Basically, he enumerated the number of different sequences of games that result in elimination of gambler after $p$ wins and $m+p$ losses and multiplied it to the probability of a particular sequence of $p$ wins and $m+p$ losses. Then he added up what he found for all nonnegative 
integer values of $p$. He also used the ratio test to show when the infinite series converges. According to Hofmann (1995), using some expansions and recursion formula, Ampère showed that the gambler will inevitably be ruined eventually if the odds of winning the game are less than the odds of losing. It is the same result as what we see in Section 8.3 for the case where the adversary has infinite fortune. Unlike other people, Ampère looked at this problem only for the case where the gambler can play infinitely unless he loses all his money.

\subsection{Modern method}

The Gambler's ruin probability can be obtained by the method of particular solutions because it can be described as a second-order homogeneous linear difference equation with two boundary conditions (Feller, 1970). Recall the Feller's description in Section 2.4. Define $q_{z}$ to be the Gambler's ruin probability with initial capital $z$. Then we get a difference equation,

$$
q_{z}=p q_{z+1}+q q_{z-1}
$$

with $q_{0}=1$ and $q_{a}=0$.

Suppose $p \neq q$. (6.1) admits two particular solutions $q_{z}=1$ and $q_{z}=(q / p)^{z}$. For arbitrary constants $\mathrm{A}$ and $\mathrm{B}$,

$$
q_{z}=A+B\left(\frac{q}{p}\right)^{z}
$$

represents a formal solution of (6.1). Using the two boundary conditions, A and B can be determined and the solution is

$$
q_{z}=\frac{\left(\frac{q}{p}\right)^{a}-\left(\frac{q}{p}\right)^{z}}{\left(\frac{q}{p}\right)^{a}-1} .
$$

Now, suppose $p=q \cdot q_{z}=1$ and $q_{z}=z$ can be two particular solutions for (6.1) and similarly, we get the solution

$$
q_{z}=1-\frac{z}{a} .
$$

Since $z=12, a=24, p=9 / 14$, and $q=5 / 14$ in the original problem from Pascal, the probability of A's winning (same as the probability of B's ruin, $E_{12}$ in Section 3 and $P_{A}$ in Section 6.2) will be

$$
\frac{\left(\frac{p}{q}\right)^{24}-\left(\frac{p}{q}\right)^{12}}{\left(\frac{p}{q}\right)^{24}-1}=\frac{1-\left(\frac{q}{p}\right)^{12}}{1-\left(\frac{q}{p}\right)^{24}} \text {. }
$$

The odds of A's winning versus B's winning are $9^{12}: 5^{12}$.

\section{Duration of Play}

The problem of the duration of play is basically the same as the problem of Gambler's ruin, but we are interested in the probability that the play ends at the $n$th game or before in this case. The first solutions of the duration of play were given by de Montmort, Nicholas Bernoulli, and de Moivre between 1708 and 1718, mostly without proofs or with incomplete proofs. After the original gambler's ruin probability became to be known, the probability distribution of the duration of play became the main interest for research on this subject. 


\subsection{De Moivre}

The player A has $a$ counters and B has $b$ counters. Define $P_{A, n}$ to be the probability that the player A will eventually win all B's counters within $n$ games. $P_{B, n}$ is similarly defined. De Moivre was the first to discover and publish a general method for calculating $P_{A, n}+P_{B, n}$, which is the probability that the play terminates within $n$ games. The results were contained in De Mensura Sortis (De Moivre, 1711) and Doctrine of chances (De Moivre, 1718). By Thatcher (1957), for the case where $a$ is infinite and $n-b$ is odd, he found

$$
P_{B, n}=\text { first } \frac{1}{2}(n-b+1) \text { terms of }(p+q)^{n}+\text { first } \frac{1}{2}(n-b+1) \text { terms of }\left(\frac{p}{q}\right)^{b}(q+p)^{n} .
$$

He published the above result with a similar one for $n-b$ even without proof. He also gave a solution for $P_{A, n}+P_{B, n}$ when both $a$ and $b$ are finite. Later, de Moivre discovered the asymptotic form for $P_{A, n}$ when $a=b$ and $p=q$.

\subsection{De Montmort and Nicholas Bernoulli}

In 1710, de Montmort found a method for calculating $P_{A, n}$ and $P_{B, n}$ separately for the case $p=q$. He sent some numerical results to John Bernoulli on 15 November, 1710 and John Bernoulli passed it to his nephew Nicholas Bernoulli. Nicholas Bernoulli sent back to de Montmort the general solution of $P_{A, n}$ and $P_{B, n}$ for $p \neq q$ without proof. He also confirmed his answer by checking if it gives a correct probability of Gambler's ruin as $n$ goes to infinity. The solution was published by de Montmort in 1713 in Essay d'Analyse sur les Jeux de Hazard(Analytical Essay on Games of Chance) (De Montmort, 1713), second edition (Thatcher (1957) or Hald (1990) for details).

\subsection{Use of inhomogeneous difference equation (Feller, 1970)}

Assume that the duration of the game has a finite expectation $D_{z} . D_{z}$ is the expected duration of game when the gambler starts with $z$ dollars (or counters). Obviously,

$$
D_{z}=p D_{z+1}+q D_{z-1}+1,
$$

for $0<z<a$ with $D_{0}=0$ and $D_{a}=0$.

Suppose $p \neq q . z /(q-p)$ is a formal solution for (7.1). Since any difference of two solutions of (7.1) should be the solution for (6.1), for arbitrary constants A and B,

$$
D_{z}=\frac{z}{q-p}+A+B\left(\frac{q}{p}\right)^{z}
$$

is a formal solution of (7.1). Using the two boundary conditions, we get

$$
D_{z}=\frac{z}{q-p}-\frac{a}{q-p} \times \frac{1-\left(\frac{q}{p}\right)^{z}}{1-\left(\frac{q}{p}\right)^{a}} .
$$

If $p=q, D_{z}=-z^{2}+A+B z$ will be a formal solution. Using boundary conditions, the expected duration in case of $p=q$ is

$$
D_{z}=z(a-z)
$$

We can also get the same result if we let $p \rightarrow 1 / 2$ in (7.2). 


\subsection{Generating functions}

Using generating functions, we can obtain the probability that the gambler is ruined at the end of $n$th game as well as the probability that the gambler is eventually ruined or the expected duration of play. To get the explicit expression, the method of partial fraction expansion is generally used. In his books, Doctrine of Chances (Moivre, 1738, 1756), de Moivre studied the partial fraction expansion of a generating function in this perspective. In 1776, Laplace derived the probability that the play terminates within $n$th game but it was not complete. The explicit formula for the probability of ruin at the $n$th game goes back to Lagrange, 1777. For more details, one can refer to Fieller (1931).

\section{Applications}

In this section, we consider several types of applications of the Gambler's ruin problem. For the contents of this section, we mainly refer to Feller (1970).

\subsection{Reflecting and elastic barriers}

The classical Gambler's ruin problem can be described as a one-dimensional random walk with two absorbing barriers. We can think of different kinds of barriers, too. Typical examples are reflecting barriers and elastic barriers. Again, a particle is moving along $x$-axis starting at point $0<z<a$. Suppose 1 is a reflecting barrier. Then whenever the particle is at 1 , it has probability $p$ of moving to 2 and probability $q$ to stay at 1 . In gambling terminology, whenever the gambler loses his last dollar (or counter), it is replaced by his opponent so that the game can continue. If there are two reflecting barriers, the game never stops. Now, suppose 1 is an elastic barrier. From position 1, the particle moves to 2 with probability $p$, stays at 1 with probability $\delta q$, and is absorbed to 0 with probability $(1-\delta) q$ for a given value of $0<\delta<1$. In gambling situations, whenever the gambler loses the last dollar(or counter), he is getting a chance to toss a coin which lands heads with probability $p$. If the coin lands heads, the last dollar is replaced by the opponent. Otherwise, he bankrupts. An elastic barrier with $\delta=1$ is a reflecting barrier, and one with $\delta=0$ is an absorbing barrier.

\subsection{Changing stakes}

Let us consider the effect of changing stakes on the ruin probability and the expected duration of the game. Note that changing unit from a dollar to a half dollar is equivalent to double the initial capital of the two players. Define $q_{z}^{*}$ to be the gambler's ruin probability with the initial capital $z$ when he is betting a half dollar each time. Then from (6.2),

$$
q_{z}^{*}=\frac{\left(\frac{q}{p}\right)^{2 a}-\left(\frac{q}{p}\right)^{2 z}}{\left(\frac{q}{p}\right)^{2 a}-1}=q_{z} \frac{\left(\frac{q}{p}\right)^{a}+\left(\frac{q}{p}\right)^{z}}{\left(\frac{q}{p}\right)^{a}+1},
$$

where $q_{z}$ is the gambler's ruin probability with a dollar stakes. It is easy to see that if $q>p, q_{z}^{*}>q_{z}$ and otherwise, $q_{z}^{*} \leq q_{z}$. It means that if a game with constant stakes is unfavorable to the gambler, to select the stake as large as possible minimizes the probability of eventual ruin.

Expected duration of game with half stakes can be calculated using the explicit expression of $D_{z}$ in Section 7.3. An interesting phenomenon is that if the stake is changed to a half, then the expected duration becomes more than double (not just a double). If we can play for one hour in a casino with initial money $a$, then we can expect to play for more than two hours with initial money $2 a$. Let us show this using Wald's theorem as follows. Define $N$ to be the duration of the game and $X_{i}$ to be 
1 if the gambler wins in the $i$ th game and -1 otherwise. Then $X_{i}$ 's are independent and identically distributed and the gain of the gambler is $X_{1}+X_{2}+\cdots+X_{N}$ if the stake is 1 . On the other hand,

$$
E(\text { gain })=(a-z)\left(1-q_{z}\right)-z q_{z}=a\left(1-q_{z}\right)-z \text {. }
$$

By Wald's theorem,

$$
E(\text { gain })=E\left(\sum_{i=1}^{N} X_{i}\right)=E(N) E(X)=D_{z}(p-q),
$$

since $N$ is a stopping time for $X_{1}, X_{2}, \ldots$ Now, change the stake to a half. Then

$$
E^{*}(\text { gain })=E\left(N^{*}\right) E\left(X^{*}\right)=\frac{p-q}{2} D_{z}^{*},
$$

where $*$ stands for the random variable with half stakes.

$$
\begin{aligned}
E(\text { gain })-E^{*}(\text { gain }) & =\frac{p-q}{2}\left(2 D_{z}-D_{z}^{*}\right)=\frac{q-p}{2}\left(D_{z}^{*}-2 D_{z}\right) \\
& =a\left(1-q_{z}\right)-z-\left(a\left(1-q_{z}^{*}\right)-z\right)=a\left(q_{z}^{*}-q_{z}\right) .
\end{aligned}
$$

The third equality comes from (8.1). Therefore,

$$
D_{z}^{*}-2 D_{z}=\frac{2 a}{q-p}\left(q_{z}^{*}-q_{z}\right)
$$

It is easy to see that if $p \neq q, D_{z}^{*}$ is greater than $2 D_{z}$. It is true for the case where $p=q$ and it can be checked from the explicit expression of $D_{z}$.

\subsection{Limiting case}

The limiting case $a=\infty$ corresponds to a game against an infinitely rich adversary who is willing to play infinitely. It may not be a realistic situation in a game between two individual players, but if we play gambling in a big casino, we are, in fact, playing with an infinitely rich adversary. This situation may be more natural in a random walk situation: A particle is moving along the $x$-axis starting at $z>0$, and there is only one absorbing barrier at $0 . q_{z}$ is the probability that the particle ever reaches the origin. Let $a \rightarrow \infty$ in (6.2) and (6.3) to get the gambler's ruin probability in the limiting case,

$$
q_{z}= \begin{cases}1, & \text { if } p \leq q, \\ \left(\frac{q}{p}\right)^{z}, & \text { if } p>q .\end{cases}
$$

If $p \leq q$ which means that the game is unfavorable to the gambler, then he will eventually be ruined. But if $p>q$, then there is a positive probability that he will never be ruined even when he is playing with an infinitely rich opponent. It is obvious that if the gambler has more money initially or $p$ gets larger, then the Gambler's ruin probability will get smaller.

To see the effect of $a=\infty$ on the expected duration, let $a \rightarrow \infty$ in (7.2) and (7.3). We can see that if $p<q$, then $D_{z} \rightarrow z /(q-p)$ and otherwise, it goes to infinity. Since the opponent has infinite fortune, the game may go on forever even though the game is favorable to the gambler. If the game is unfavorable to the gambler, then the game is expected to stop after some finite time. As we can easily expect, if the gambler's initial fortune gets larger, or $q-p$ gets smaller, (two players' skill is not very different) then the expected duration will get longer. 


\subsection{Connection with diffusion processes}

In a gambling situation, it is not sensible to think of infinitesimal amount of stake or infinitesimal time interval between games. But in a random walk situation, we can imagine very small length of individual steps and very small jump size. If the jump size goes to 0 and the number of jumps in a unit time goes to $\infty$ in an appropriate manner, then the random walk converges to a continuous motion such as Brownian Motion process or other diffusion processes.

There are more applications such as multi-dimensional random walk or sequential sampling. Readers can refer to Feller (1970).

\section{Conclusion}

After it had been proposed by Pascal to Fermat in 1656, the Gambler's ruin problem has been widely studied among leading scholars for more than a hundred years. The first solution was given by Pascal himself and Fermat, but there exist no proof or idea about how they ended up with their solutions. Huygens introduced the problem and provided the solution to the public by including them in his publication in 1657, and later gave the first description of a proof. James Bernoulli found a solution for a general situation, but he did not give any proof. In 1711, the generalized solution was finally proved in a simple and elegant way by de Moivre. Since then, the research became more concentrated on the problem of the duration of play. Many mathematicians such as de Moivre, de Montmort, Nicholas Bernoulli, Laplace and Lagrange studied the duration of play in $18^{\text {th }}$ century. In 1777 , Lagrange achieved the explicit formula for the probability of gambler's ruin at the $n^{\text {th }}$ trial using generating functions.

Besides, the Gambler's ruin is a beginning of the theory of random walk and diffusion processes. The method of difference equation which was widely used for solving this problem can be considered as an introduction to the differential equation of diffusion theory. Diffusion processes play a very important role in modern finance theory in the sense that underlying asset price processes are many times modeled as diffusion processes. Although the Gambler's ruin problem may not be applied directly to any problem of modern finance, it is not hard to see how the modern finance theory is developed from the classical gambling situation.

\section{References}

Ampère, A.-M. (1802). Considérations sur la Théorie mathématique du Jeu, Lyon Chez les frères Perisse.

Bernoulli, J. (1713). Ars Conjectandi, Thurnisius, Basilea. Reprinted in Editions Culture et Civilisation, Bruxelles, 1968, and in Die Werke von Jakob Bernoulli, 3, Birkhäuser, Basel, 1975.

Edwards, A. W. F. (1987). Pascal's Arithmetical Triangle, Griffin, London.

Feller, W. (1970). An Introduction to Probability Theory and Its Applications, 3rd Ed., Wiley.

Fieller, E. C. (1931). The duration of play, Biometrika, 22, 377-404.

Hald, A. (1990). A History of Probability and Statistics and Their Applications before 1750, Wiley.

Hofmann, J. R. (1995). André-Marie Ampère: Enlightenment and Electrodynamics, Cambridge University Press.

Huygens, C. (1657). De Ratiociniis in Ludo Aleae, printed in Exercitationum Mathematicarum by F. van Schooten, Elsevirii, Leiden. Reprinted in Oeuvres, 14, (1920).

Moivre, A. de (1711). De Mensura Sortis, seu, de Probabilitate Eventuum in Ludis a Casu Fortuito Pendentibus, Philosophical Transactions, 27, 213-264. 
Moivre, A. de (1718). The Doctrine of Chances: or, A Method of Calculating the Probability of Events in Play, Pearson, London.

Moivre, A. de (1738). The Doctrine of Chances, second edition, Woodfall, London.

Moivre, A. de (1756). The Doctrine of Chances, third edition, Millar, London.

Montmort, P. R. de (1713). Essay d'Analyse sur les Jeux de Hazard, Seconde Edition. Revûe et augmentée de plusieurs Lettre. Quillau, Paris.

Ore, O. (1960). Pascal and the intervention of probability theory. American Mathematical Monthly, 67, 409-419.

Ross, S. (1997). A First Course in Probability, 5th Ed. Prentice-Hall.

Struyck, N. (1716). Calcul des Rentes viagères, Reprinted in Oeuvres (1912), 194-210.

Thatcher, A. R. (1957). A note on early solutions of the problem of the duration of play, Biometrika, 44, 515-518.

Received March 15, 2013; Revised March 27, 2013; Accepted March 27, 2013 